\title{
Impact of Language Diversity and Social Interaction on Knowledge Transfer
}

\author{
Chavi Fletcher-Chen \\ IESEG School of Management (LEM UMR CNRS 9221), Université Catholique de Lille, Lille, France
}

\begin{abstract}
This research calls attention to how language diversity and social interaction play a pivotal role in influencing the aspects of absorptive capacity and communication for knowledge transfer. The multinational corporation (MNC) is a multilingual community where culture is a given but language itself is a choice in communication. While MNCs are geographically dispersed organizations, the social interaction at the team level is based on the physical proximity and the channels of computer-medicated communication (CMC). This research has conducted a field study within the information technology (IT) industry, including 296 product design and sales service teams from 115 MNC foreign subsidiaries based in formerly colonized countries in Southeast Asia. The results partially support the conceptual model and demonstrate an impact of language diversity and social interaction on knowledge transfer. These findings contribute to the understanding of the underlying mechanisms of knowledge transfer and how product design and sales service teams in particular cope with the challenge of language diversity and condition of social interaction.
\end{abstract}

Keywords: team, language, absorptive capacity, communication, knowledge transfer

\section{Introduction}

Multinational corporations (MNCs) are exposed to a complex structure due to the presence of its subunits dispersed globally. The multilingual resources and similar regional Asian culture in Asian emerging markets create a competitive advantage for MNCs to expand their market. While organizations perform different functional activities (such as marketing and technological know-how), teams providing specific functional knowledge integrate functional activities. Studies on teams for collaborative work in organizations have typically found positive outcomes, especially that teams in product design and sales service have become a vital component in MNCs to meet global market demands as they provide more perspectives and information and exert more effort (e.g., Bandow, 2001; Strubler \& York, 2007). Furthermore, teams can be proximately accessible or dispersed geographically to overcome the "transmission losses" between individuals that occur during the transfer of complex procedural knowledge (Mudambi, 2002). While knowledge transfer in collaborative work involves information sharing and requires knowledge competencies to accomplish a task, effective communication facilitates knowledge transfer.

Minbaeva (2007) highlighted two essential factors that challenge the success of knowledge transfer, namely, the characteristics of the individuals involved in the knowledge transfer process and the characteristics

Chavi Fletcher-Chen, Ph.D., assistant professor, IESEG School of Management (LEM UMR CNRS 9221), Université Catholique de Lille. 
of that knowledge. The process of knowledge transfer becomes even more challenging due to the difficulties in communicating in a multilingual community. This is due to two main reasons. Firstly, the multiple language choices complicate communication due to the implication of language choice. Team actors adapt to the complexity of pragmatic language choice, i.e., context for work operation and knowledge expertise and closeness of common ground and local environment, to create shared sense making for knowledge transfer. Secondly, knowledge transfer is embedded in social interactions. The social interaction under the structure of communication settings may cause knowledge stickiness due to communication bandwidth and synchrony (Noorderhaven \& Harzing, 2009) in transferability of information, knowledge, and expertise.

Geographically dispersed teams operating in MNCs confront the needs to integrate and coordinate activities for internal knowledge movement and dynamic global market. The enabler of computer-mediated communication (CMC) facilitates communication across functional specialty and geographical boundaries to enhance coordination of multinational activities (Andersen \& Foss, 2005). How these dispersed teams adapt different communication combination settings, i.e., CMC and/or face-to-face (F2F), to integrate collaboration manifests the structure of their social interactions. The subjects in past studies relating social factors mostly focus on productivity gains as task performance (Dennis, Fuller, \& Valacich, 2008), instead of focusing on the social structures created by the combination of CMC and F2F settings. In addition, these studies focused on the outcome between the levels of the individual and the organization (e.g., Felin \& Foss, 2005; Felin, Foss, Heimeriks, \& Madsen, 2012; Cohen \& Levinthal, 1990). However, the study of knowledge transfer at the team level in MNCs has received little attention.

This research highlights the gap of MNC knowledge transfer between geographically dispersed teams. The combination of communication channels among these teams forming different levels of social interaction may affect how they perform their communication and their ability for knowledge transfer. Also, teams combining different knowledge specialties under the institutional condition of MNCs constitute individuals from various countries making of a multilingual community. MNCs can simply use a corporate lingua franca (LF) as a shared language to bridge language differences for communication. Yet, when team actors have multiple languages in common, their choice of language may serve different purposes. Communicating in a single LF or in multiple languages becomes a choice. Hence, the choice of language may have an impact on communication and ability to transfer knowledge.

This research utilizes the MNC subsidiaries based in formerly colonized Southeast Asian countries, e.g., Taiwan colonized by Japan, and Singapore, Hong Kong, and Malaysia colonized by the UK. The product design and sales service teams in these countries share a similar cultural background and are immersed in a multilingual environment. These dispersed teams can take an advantage of this colonization in language to reduce communication problems and incorporate regional social-cultural knowledge to facilitate the communication among their regional organizational units. Although past research has evaluated communication factors of collaborative teams (e.g., Lowry, Roberts, Romano, Cheney, \& Hightower, 2006), an obvious condition of language diversity has received little attention. This paper aims to contribute to knowledge by identifying the impact of multilingual diversity and social interaction on knowledge transfer within the context of MNC's operating in emerging markets.

In the next section, the literature review develops the concepts of language diversity in MNCs and social interaction under various communication settings from theories, and then, predicts the impact of language 
diversity and social interaction on knowledge transfer. The author proceeds with the research design and a discussion of the findings and their implications for research and practice in the sections that follow.

\section{Theory and Hypotheses}

The literature review below describes the condition of language diversity in MNCs and the level of social interaction and predicts their impacts on knowledge transfer.

\section{Language Diversity in Emerging Markets}

Strategic rationality in the global language design in MNCs plays a critical role. The assumption of a multilingual community conveniently recognized that MNCs depend on a corporate LF as a shared language to enhance communication and share knowledge (Phene, Madhok, \& Liu, 2005). The critical inputs created in a global environment of MNCs also indicate a possible role for language beyond facilitating effective communications. Luo and Shenkar (2006) aligned the impact of language in MNCs and have proposed that involving multiple languages benefits the improvement of intra-unit value creation and the encouragement of intra-unit business communication and socialization.

Languages are constantly evolving to provide multi-directional information. Multilingual individuals must communicate to encode information, and store and retrieve that information. The information expressed through new vocabularies and language usages create new jargons and dialects to represent mixtures of diverse languages, e.g., a hybrid language or a unique form of using multiple choices of language. Hence, enhancing the characteristics of intensity and breadth through multiple languages facilitates knowledge transfer in MNCs (Luo \& Shenkar, 2006).

When there are overlapping native, local, and corporate languages, languages can have an impact on knowledge transfer and diffusion due to organizational management in operation (Vaara, Tienari, \& Piekkari, 2005) and pragmatic choices of language across knowledge boundaries (Carlile, 2004). For example, people from Hong Kong use Cantonese while the traditional Chinese is the same written language used in Taiwan. Singapore and Taiwan use Mandarin as well as Taiwanese, while Singapore, Malaysia, and Hong Kong use English as a unified language due to their former colonization. Their diverse language environments benefit their communication with their foreign, local, and regional markets.

\section{Social Interaction Structured by Communication Channels}

Watson-Manheim and Bélanger (2007) suggested that communication media affect communication repertoires. When teams are dispersed in various locations, their communication media vary with the level of electronic dependence and geographic dispersion and form a structure of their social interaction. When team actors articulate their perspectives in order to make sense to others, their expressions and behavior are amplified through social interaction.

Various forms of communication described social presence to posit interactions in F2F and CMC settings. Media synchronicity theories (Dennis et al., 2008) and media richness theories (Trevino, Webster, \& Stein, 2000) distinguish the level of richness by lean media and rich media. Lean media include the use of text-based communication, e.g., e-mail, instant message, and online discussion board, while rich media include the use of synchronous media through audio and video conferencing.

Based on media theories, the level of media richness (Lengel \& Daft, 1989) involving non-verbal communication, proximity, and physical appearance can affect the level of social interaction. The interaction 
level under the restriction of communication tools, the amount of informational value provided by those tools, and communication synchronicity (Kirkman \& Mathieu, 2005) may affect how teams develop their tasks and manage their communication. Noorderhaven and Harzing (2009) distinguished two characteristics of rich communication media to facilitate social interaction for knowledge movement: bandwidth and synchrony. Bandwidth refers to the ability to directly convey and observe non-verbal and visual cues (Daft, Lengel, \& Trevino, 1987), while synchrony refers to the ability to provide and attain immediate feedback (Dennis et al., 2008).

Based on social presence theories, Short, Williams, and Christie (1976) classified communication media and their social impacts as a construct of social presence. The authors categorized the level of social interaction by identifying that communication media differ based on the quantity of transmitting non-verbal communication and the quality of converging mutual understandings. Performing proximity can present interpersonal intimacy and the need for immediacy, while physical appearance and visual contact can enhance personal awareness. This study adopts a more explicit definition of social presence as the degree to which communication media allow group actors having social presence to perceive (sense) the actual presence of the communicators and the consequent appreciation of an interpersonal relationship (Kock, 2004).

\section{Knowledge Transfer}

Knowledge transfer is at the heart of communities of practice concept (Buckley \& Carter, 2004) that builds on the notion that groups of individuals tend to reduce the barriers to knowledge absorption through social interaction and exchange. The broad dimension of knowledge involves communication and embodied transfer (Buckley \& Carter, 1999). Communication refers to interpersonal communication and information presentation. The embodied transfer relates to complex forms of knowledge carriers, such as technical knowledge in information technology (IT) products. Knowledge transfer in this research for task performance involves the cognitive prospective of work interdependency and the social aspect of communication emerging through interactions concerning task related matters as part of conducting their ongoing work.

\section{Communication}

The scope of communication in knowledge transfer is based on group communication quality (Lowry et al., 2006) and refers to how teams perceive their own team discussion effectiveness and development. Evidence indicates that the antecedents of effective communication include quality (Burgoon, Bonito, Ramirez, Kam, Dunbar, \& Fischer, 2002), openness (Lauring \& Selme, 2012; Cadiz, Griffith, \& Sawyer, 2009), richness (Luo \& Shenkar, 2006), and accuracy (O’Reilly, 1982; Gurtner, Tschan, Semmer, \& Nägele, 2007), as explained below.

Discussion quality. Discussion quality combines the evaluation levels of effectiveness and satisfaction from team actors about their communication process during discussion and discussion development (Burgoon et al., 2002). Team actors sharing and learning different perspectives allows them to jointly define the issue and/or successfully identify the problem.

Richness. Richness in communication refers to the intensive and in-depth exchange of on-topic, detailed responses and clear messages within the group (Burgoon et al., 2002) and provides apprehensive information to spread and facilitate group coordination (Luo \& Shenkar, 2006; Gurtner et al., 2007).

Openness. Openness in communication is the willingness of a set of people bound together through common interest to be receptive to the communication of others (Lauring \& Selme, 2012; Cadiz et al., 2009). 
With the goals of open communication and exchange and retention of useful and relevant information, team actors can better evaluate threats to resolve problems. Openness encourages people to express themselves for a mutual understanding and explore their own ideas, the group's perceptions of their ideas, and the ideas of others in the group (Lowry et al., 2006).

Accuracy. Accuracy is the degree to which team actors exchange information and communicate to understand each other properly and correctly (O’Reilly, 1982). Team actors adequately communicating a complex message can understand equivocality and clarify uncertainty immediately (Daft et al., 1987). The level of accuracy logically affects the quality of decisions and the amount of time required to identify issues.

\section{Absorptive Capacity}

Absorptive capacity describes the ability of a unit in the form of a person, team, or organization to use past experiences to increase the ability and learn to create knowledge and apply new knowledge (Cohen \& Levinthal, 1990; Thompson, 2011). Research on absorptive capacity has focused on a single level of organization and the level between individual and organization (e.g., Felin \& Foss, 2005; Felin et al., 2012). Szulanski’s (1996) studies focused on a best practice of knowledge transfer at the organizational level while continuous social interactions at the individual level increase knowledge sharing and subsequently reduce stickiness of knowledge transfer.

Past studies have revealed that absorptive capacity influences performance by enhancing integration of individual knowledge (Tiwana \& McLean, 2005). Teams under the multilingual and geographical conditions of MNCs may enhance the impacts of language diversity (T. Ambos \& B. Ambos, 2009) and different levels of social interactions on absorptive capacity. Griffith, Sawyer, and Neale (2003) argued that the analysis of absorptive capacity (Cohen \& Levinthal, 1990) relates to how information is noticed and how individuals in group decode and transform knowledge, and then, integrate the information and knowledge into the group's knowledge. Through the processes of assessment, assimilation, and application, individuals coordinate knowledge and transform new knowledge into useable knowledge (Cadiz et al., 2009).

Assessment. Assessment is a process to gather information and knowledge directly and indirectly from different sources and activities. The assessment process identifies issues from past experiences to help reduce overwhelming amounts of information and regulate useful information and thereby, filtering through valuable knowledge.

Assimilation. Assimilation is a process where information and knowledge collide to become meaningful. The assimilation process cognitively organizes acquired knowledge to converse new knowledge into useable knowledge and thereby makes it accessible for future use. Through analysis, processing, transformation, and interpretation of knowledge, assimilation identifies the gap between the required knowledge and the existing knowledge. Hence, meaningful knowledge creates the value for future use.

Application. Application is a process that can involve certain levels of creativity by integrating knowledge as interrelated fields of knowledge, performing an ability to recognize a situation and develop ideas-how, where, and when knowledge can be exploited. Application capability allows taking the assimilated and transformed knowledge to exploit the new knowledge in a unique way.

These constructs develop a conceptual model, as shown in Figure 1. 


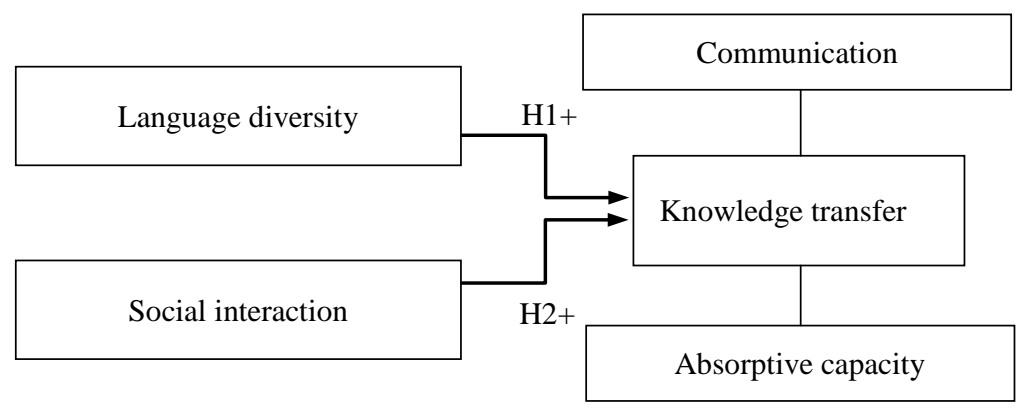

Figure 1. Conceptual model.

\section{Prediction Based on Language Diversity}

Teams share common objectives and are socially constructed through collaborative efforts and dialectically exchange different perspectives to perform knowledge. Lacking social knowledge may affect how team actors relate to others by understanding behaviors, avoiding conflict, or comprehending implicit subtleties (Levin \& Cross, 2004). Their social knowledge embedded in their cultural relatedness and social indicators form a shared mental model for knowledge transfer.

The context of MNC's operating in Southeast Asia involves the common aspects of shared languages, experiences of colonization, and cultural norms. Team actors originated from a common cultural background and sharing the same native language can easily absorb thoughts, perception, and action from others. Also, sharing the same native language empowers the team actors to communicate in a natural language, as essential for sense making in the social constitution of knowledge, and facilitates the process of understanding (Vaara et al., 2005).

MNCs rely on a corporate LF to govern a standard form of policy to solve language barriers for information flow and process development. When a corporate LF is a foreign language, applying the syntactic forms of one language to the words of another may not imply the same meaning as intended. The process of translating the full richness of meaning from one language to another is difficult. Due to the unawareness of the associations of the alternatives offered, the difficulties to cope with language distance (Dow \& Karunaratna, 2006) reduce the richness to express social subtleties of meaning. Also, lacking the richness in communication may explain how the complexity of knowledge is likely to create knowledge stickiness.

Under the complexity of multilingual community, individuals within teams can be from different nations and/or the same nation. Team actors from different nations may speak different native languages and a corporate LF can bridge the communication gap. Yet, team actors from different nations may have other shared language(s) to bridge the communication (e.g., gaps that overseas Chinese living in Southeast Asian countries using Chinese languages). Team actors from the same nation will share a common language, which can be local, native, and/or national language. The number of language choices suggests two situations of communication: (a) using a corporate LF as a single language choice; and (b) combining different languages as multiple language choices.

To overcome unexpected confusion and avoid misinterpretation, multiple language choices may convey richness and provide accuracy. Multilingual team actors may pragmatically apply the associated language to its type knowledge. For example, Singaporean team actors interacting with other overseas Chinese choose a common Chinese language in association with a social or a cultural context, i.e., favoring to maintain social interaction while using a corporate language (English) as an operating tool to execute organizational standards 
and practice their specialty. Communicating in multiple languages to associate different contexts may suggest an effort of pooling information and behavioral engagement to enhance social interaction intensity.

A multilingual environment allows team actors leveraging competencies according to the choice of communicating in a single LF or in multiple languages, to enhance the process of knowledge transfer. The previous literature leads to the hypotheses $1 \mathrm{a}$ through $1 \mathrm{~g}$.

Hypothesis 1 (H1): Teams communicating in multiple languages can better enhance capacity in (a) assessment, (b) assimilation, and (c) application; and perform (d) greater communication openness, (e) more accurate communication, (f) richer communication, and (g) a higher quality of discussion than the teams communicating in a signal LF.

\section{Prediction Based on Condition of Social Interaction}

The knowledge transfer can be derived from the combination of knowledge absorption and communication for rules of action. This research reintegrated a component of value identification defined by Cohen and Levinthal (1990) and recommended by Todorova and Durisin (2007) and applied the model suggested by Zahra and George (2002) to identify the coordination characteristics of absorptive capacity. To integrate team collaboration, teams enhance their capacity by absorbing diversity and communicate for sense making by adapting to different levels of social interaction. To align with their interactivity and maintain bi-directional dynamics, the level of social interaction and the choice of language could create a different platform to promote knowledge transfer.

Social presence theory states that lacking sufficient social presence may not benefit interactivity and reciprocity between communication sender and receiver (Short et al., 1976). Social cues enable communicators to perceive one another and influence the message interpretation and judgment. The level of social presence through CMC can de-individualize communicators for their social interaction (Roberts, Cheney, Sweeney, \& Hightower, 2005). A meta-analysis of 4,795 groups revealed that information as openness sharing positively predicted knowledge transfer (Mesmer-Magnus \& DeChurch, 2009). These studies suggest that deep level information becomes available through verbal and non-verbal interactions and value congruity would lead to increased communication accuracy. The greater social presence of a medium can covey, the greater immediacy and warmth of the communication performs.

Nonaka and Takeuchi (1995) emphasized that knowledge transferring can be better conducted via a more direct and natural routine for both socialization and internalization. Noorderhaven and Harzing (2009) indicated that communication intensity and bandwidth enhance social interaction, and argued that social interactions under rich communication media facilitate the movement of knowledge. The communication setting that provides wider bandwidth and better synchrony allows parallel information for simultaneous interactions. Cadiz et al. (2009) suggested that F2F setting can provide more engaging opportunities for sense making, facilitation, and deep discussion, perform affection that signals a person's reaction to a message, as well as encourage the counterpart react to the message, i.e., explicit work products, technological expertise, and social aspects of tacit knowledge. CMC setting can result in choices to perform more direct forms of communication and provide a more presentable and amenable information to transfer knowledge (Tidwell \& Walther, 2002; DeSanctis \& Poole, 1994). Although the CMC that incorporates visual contact and physical appearance can increase a mutual sense of shared space, the absence of social cues in CMC de-individualizes actors to relate to others. Lean CMC limits the level of synchrony of interaction, reducing social cues or gestures common to 
communication and also obstructing awareness of a communicator's physical appearance. This diminishes the group awareness of the self and the other. Combining F2F and CMC settings provides a wider bandwidth and effective synchrony (Barner-Rasmussen \& Bjorkman, 2005) than one setting of interaction style.

Supportive research illustrates that a lower level of social presence results in fewer cues and reduces the opportunities to generate interaction and reciprocity, accordingly diminishing communication quality (Roberts et al., 2005). A physical distance created in CMC can generally focus on the reciprocity of presentable information or objects whereas the social setting among actors becomes more symbolic. The flexibility of CMC overcomes geographic boundaries and allows transmitting synchronous information or presenting asynchronous information that can be stored and retrieved (Dennis et al., 2008). Also, team actors may be unaware as to the activity of others or if they are conducting work and how committed to the engagement. Accordingly, team actors may be less involved in open communication and less motivated to participate in team collaboration. When team actors do not expose their behavior sufficiently for interactivity and develop tacit norms effectively, social knowledge can become sticky and difficult to be assessed and absorbed. Hence, the performance of knowledge transfer may reduce along with the level of social interaction.

Media theories and social theories regard that communication media conveying more cues would lead to greater social interaction. The author expects that a higher level of social interaction is likely to have a positive effect on knowledge transfer. The proposition of communication conditions for social interaction leads to hypotheses 2a-2g.

Hypothesis 2 (H2): Teams with a higher level of social interaction can better enhance capacity in (a) assessment, (b) assimilation, and (c) application; and perform (d) greater communication openness, (e) more accurate communication, (f) richer communication, and (g) a higher quality of discussion than the teams with a lower level of social interaction.

\section{Methods}

\section{Respondents and Procedure}

The hypotheses were tested in an IT industry, in which knowledge changes quickly. The targeted teams were the product design and sales service teams, who are responsible for designing complex system software and hardware solutions and selling products to other organizational customers. Knowledge within this type of team revolves around the subjects, such as operating systems requirements, hardware equipment, programming language, and customer demands for the interfaces. Team task is to focus on the goal of technical sophistication resulting in high quality system design, sales, and customer feedback in a competitive environment where technology requires rapid change and provides customers with instant feedback. Team actors interact to design technical solutions that combine the various specializations and technical sophistication to provide an integrated system.

Prior to administration, a pilot study tested the survey instrument with three groups of individuals, academic experts, and professionals in an area of international management. This research was initially conducted in 115 MNC foreign subsidiaries in Southeast Asian countries, namely, Taiwan, Hong Kong, Malaysia, Singapore, Philippine, and Indonesia. Through the generous support of teams in those subsidiaries, the questionnaire survey was also distributed to other product design and sales service teams in their local subsidiaries. The collected data focused on the countries that were colonized and are influenced by a similar regional culture (Confucianism). MNCs in formerly colonized countries share a common aspect on the 
adaptation of multiple languages, amplifying the concept of multilingual communities. The corporate language used in those subsidiaries is a common business language (English) for organizational operation while IT jargons are originated from English. Thus, the local or national language in these subsidiaries is different from the corporate language. After deleting those responses with substantial missing data, the finished sample consisted of 296 teams, which represents a response rate of 38\% while team size varies from 5 to 12 ( $M=7.5$; $S D=1.95)$.

The data were collected from the teams where the local language has a high level of linguistic distance and is from different language origins (West \& Graham, 2004; Dow \& Karunaratna, 2006). The survey language is English. The following information comprises the demographic data of the respondents in this research: (a) age ranging from 25 to 48, yielding an average of 32 years $(S D=4.62)$; (b) team composition of nationality including single nationality composition (38\%) and various nationality compositions (62\%); and (c) the mother tongue within the teams involving a shared native language (47\%) or different native languages (53\%).

\section{Measures}

The general information in this questionnaire survey requests team leaders to reflect a recent finished teamwork of designing and selling complex software and hardware solutions to other organizations and identify their communication settings. To examine how language diversity and social interaction impact on knowledge transfer, identifying communication settings and language diversity in the beginning of the questionnaire helps frame the independent variables in terms of language diversity and social interaction. The dependent variables constructed in knowledge transfer in this study are group communication construct and absorptive capacity.

Language diversity. Through the categorical measurement in this questionnaire survey, language diversity identifies the number of language choices. The number of languages categorizes the choice of communicating in a single LF or in multiple languages.

Social interaction. Communication studies have differed CMC from F2F setting (Rice \& Gattiker, 2001; D’Urso \& Rains 2008). The communication categorizes three settings of social interaction, which are: (a) low level of social interaction - virtual with high frequency of lean CMC over rich CMC; (b) a medium level of social interaction-virtual with high frequency of rich CMC over lean CMC; and (c) a high level of social interaction-proximate with CMC and F2F. The features of F2F and CMC increase the level of social presence by virtue of increasing media richness. Table 1 overviews the social interaction that frames each condition.

Table 1

Number of Teams Based on Social Interaction Across Language Diversity

\begin{tabular}{lllll}
\hline Interaction diversity & $\begin{array}{l}\text { Low: Virtual emphasizing } \\
\text { lean CMC }\end{array}$ & $\begin{array}{l}\text { Moderate: Virtual } \\
\text { emphasizing multiple CMC }\end{array}$ & $\begin{array}{l}\text { High: Proximate with CMC } \\
\text { and F2F }\end{array}$ & Total \\
\hline Single LF & 50 & 38 & 51 & 139 \\
Multiple languages & 58 & 51 & 48 & 157 \\
Total & 108 & 89 & 99 & 296 \\
\hline
\end{tabular}

Notes. ${ }^{1}$ Virtual with high frequency of lean CMC over rich CMC; ${ }^{2}$ Virtual with high frequency of rich CMC over lean CMC; and ${ }^{3}$ High F2F interactions with multiple media provided by CMC.

Knowledge transfer. The questionnaire survey (see Appendix) partially adapted communication survey scales (Lowry et al., 2006) and measurement scales for absorptive capacity (Cadiz et al., 2009). Scales adapted for the dependent variables of knowledge transfer measure three aspects of absorptive capacity in assessment 
( $\alpha=0.88$ ), assimilation ( $\alpha=0.89$ ), and application ( $\alpha=0.88)$, and four aspects of communication in discussion quality ( $\alpha=0.85)$, richness ( $\alpha=0.84)$, openness $(\alpha=0.88)$, and accuracy ( $\alpha=0.76)$ (see Table 2$)$.

Table 2

Results of Exploratory Factor Analysis ${ }^{a}$

\begin{tabular}{|c|c|c|c|c|c|c|c|}
\hline & Richness & $\begin{array}{l}\text { Discussion } \\
\text { quality }\end{array}$ & Assimilation & $\begin{array}{l}\text { Communication } \\
\text { quality }\end{array}$ & Application & Assessment & Accuracy \\
\hline $\mathrm{f} 4$ & 0.764 & & & & & & \\
\hline f3 & 0.760 & & & & & & \\
\hline f5 & 0.689 & & & & & & \\
\hline $\mathrm{f} 2$ & 0.688 & & & & & & \\
\hline f1 & 0.661 & & & & & & \\
\hline g2 & & 0.879 & & & & & \\
\hline g1 & & 0.818 & & & & & \\
\hline g3 & & 0.742 & & & & & \\
\hline g4 & & 0.602 & & & & & \\
\hline b2 & & & 0.870 & & & & \\
\hline b3 & & & 0.845 & & & & \\
\hline b1 & & & 0.793 & & & & \\
\hline $\mathrm{d} 2$ & & & & 0.911 & & & \\
\hline d1 & & & & 0.823 & & & \\
\hline d3 & & & & 0.770 & & & \\
\hline c2 & & & & & 0.877 & & \\
\hline c3 & & & & & 0.865 & & \\
\hline $\mathrm{c} 1$ & & & & & 0.756 & & \\
\hline a1 & & & & & & 0.874 & \\
\hline a2 & & & & & & 0.833 & \\
\hline a3 & & & & & & 0.821 & \\
\hline$\overline{\mathrm{e} 1}$ & & & & & & & 0.752 \\
\hline e2 & & & & & & & 0.726 \\
\hline e3 & & & & & & & 0.657 \\
\hline Cronbach's $\alpha$ & 0.840 & 0.846 & 0.886 & 0.878 & 0.884 & 0.880 & 0.756 \\
\hline
\end{tabular}

Notes. Extraction method: Maximum Likelihood; Rotation method: Promax with Kaiser Normalization; and ${ }^{\text {a }}$ Rotation converged in six iterations.

\section{Analysis}

The question items focus on team actors' responses about the team. These constructs were analyzed at the team level of analysis as each team leader gathers representative information on behalf of the team. Each scale used a 7-point Likert-type scale ranging from 1 as "Negative" anchor to 7 as "Positive" anchor. The past study identified absorptive capacity and communication separately, yet this study explores the relationships between absorptive capacity and communication by involving both cognitive and social aspects. An exploratory factor analysis with Maximum Likelihood Analysis (see Table 2) was firstly conducted to assess and validate the scales and yielded seven factors $\left(K M O=0.83 ; p^{* * *}<0.001\right)$, rotated by Promax with Kaiser Normalization with eigenvalues above 1 (explained variance: 63.73\%). The extraction of communalities is above 0.4. The factor loadings are also significant high (>0.6). This score was then incorporated into a confirmatory factor analysis. The resulting measurement model fits well, as evidenced by the fit statistics $\left(\chi^{2}{ }_{(188)}=234.89, \chi^{2} / d f=1.25\right.$, and $p=0.01 ; R M S E A=0.03, A G F I=0.91, C F I=0.99, N F I=0.93$, and $R M R=0.07)$. All loadings were high (the lowest was 0.60 ). Table 3 contains the correlation matrix of the constructs. 
Table 3

Means, Standard Deviations, and Estimated Correlations $(N=296)$

\begin{tabular}{|c|c|c|c|c|c|c|c|c|c|}
\hline & $M$ & $S D$ & Accuracy & Assessment & Application & Openness & Assimilation & $\begin{array}{l}\text { Discussion } \\
\text { quality }\end{array}$ & Richness \\
\hline Accuracy & -0.02 & 0.89 & 1 & - & - & - & - & - & - \\
\hline Assessment & -0.06 & 1.36 & -0.11 & 1 & - & - & - & - & - \\
\hline Application & -0.20 & 1.23 & -0.06 & $0.30^{* *}$ & 1 & - & - & - & - \\
\hline Openness & 0.05 & 1.28 & $0.23^{* *}$ & $-0.13^{*}$ & $0.42^{* *}$ & 1 & - & - & - \\
\hline Assimilation & -0.03 & 1.28 & -0.05 & $0.41^{* *}$ & $0.69^{* *}$ & $0.30^{* *}$ & 1 & - & - \\
\hline Discussion quality & 0.12 & 1.00 & $0.21^{* *}$ & $-0.40^{* *}$ & $-0.21^{* *}$ & -0.02 & $-0.26^{* *}$ & 1 & - \\
\hline Richness & 0.13 & 0.77 & $0.37^{* *}$ & $-0.55^{* *}$ & $-0.31^{* *}$ & $0.17^{* *}$ & $-0.26^{* *}$ & $0.44^{* *}$ & 1 \\
\hline
\end{tabular}

Notes. ${ }^{* *}$ Correlation is significant at the 0.01 level (2-tailed); ${ }^{*}$ Correlation is significant at the 0.05 level (2-tailed).

Seven factors extracted from knowledge transfer served as dependent variables, in which three factors on absorptive capacity and four factors on communication. To test Hypotheses 1 and 2, regarding the impact of language diversity and social interaction on knowledge transfer, this study conducted a repeated-measures multivariate analysis of variance (MANOVA) and subsequent post-hoc tests. Post-hoc analyses allow observing the differences specifically within each dependent variable. The MANOVA results revealed explicit differences among three conditions of social interaction and two types of language diversity for each of the dependent variables. The primary analysis involved a two-way ANOVA to look for interactions between the two independent variables as well as how much of an influence each of the independent variables has had on their own.

\section{Results}

The MANOVA results on knowledge transfer revealed explicit differences between social interaction $\left(F_{(14,568)}=5.04 ; p^{* * *}<0.001\right.$; Wilk's $\left.\lambda=0.79\right)$ and language diversity $\left(F_{(7,284)}=8.45 ; p^{* * *}<0.001\right.$; Wilk's $\lambda=$ 0.83). Table 4 indicates the support of each hypothesis for each construct and Table 5 shows the mean results for each condition of social interaction. The interaction plots will demonstrate the figures of associated finding results in the aspects of absorptive capacity and communication.

Table 4

Summary of Hypotheses and Results

\begin{tabular}{|c|c|c|c|c|}
\hline Hypothesis & & & Measure & Support \\
\hline \multirow{7}{*}{$\begin{array}{l}\text { H1: Language } \\
\text { diversity }\end{array}$} & $1 \mathrm{a}$ & Multiple $>$ Single & Assessment & $\begin{array}{l}\text { Yes, } F_{(1,290)}=10.58, p^{* *}<0.01 \text { with a significant interaction; } \\
F_{(2,290)}=3.08, p^{*}<0.05 ; t(294)=-2.86, p^{* *}<0.01\end{array}$ \\
\hline & $1 \mathrm{~b}$ & Multiple $>$ Single & Assimilation & Yes, $F_{(1,290)}=3.91, p^{*}<0.05 ; t(294)=-1.81, p^{\dagger}<0.1$ \\
\hline & 1c & Multiple $>$ Single & Application & Yes, $F_{(1,290)}=4.85, p^{*}<0.05 ; t(294)=-2.21, p^{* *}<0.01$ \\
\hline & $1 \mathrm{~d}$ & Multiple $>$ Single & Openness & No, $F_{(1,290)}=3.59, p^{+}<0.1 ; t(294)=-1.67, p^{+}<0.1$ \\
\hline & $1 \mathrm{e}$ & Multiple $>$ Single & Accuracy & Yes, $F_{(1,290)}=4.38, p^{*}<0.05 ; t(294)=-1.86, p^{+}<0.1$ \\
\hline & 1f & Multiple $>$ Single & Richness & Yes, $F_{(1,290)}=9.42, p^{* *}<0.01 ; t(294)=-2.90, p^{* *}<0.01$ \\
\hline & $1 \mathrm{~g}$ & Multiple $>$ Single & Discussion quality & Yes, $F_{(1,290)}=4.88, p^{*}<0.05 ; t(294)=-1.96, p^{*}=0.05$ \\
\hline \multirow{7}{*}{$\begin{array}{l}\text { H2: Social } \\
\text { interaction }\end{array}$} & $2 \mathrm{a}$ & High $>$ Low & Assessment & Yes, $F_{(2,290)}=3.93, p^{*}<0.05, \mathrm{C}>\mathrm{A}\left(p^{*}<0.05\right)$ \\
\hline & $2 b$ & High $>$ Low & Assimilation & Yes, $F_{(2,290)}=7.07, p^{* *}<0.01, \mathrm{C}>\mathrm{A}\left(p^{*}<0.05\right)$ \\
\hline & 2c & High > Low & Application & No, $F_{(2,290)}=1.06, p=0.349$ \\
\hline & $2 d$ & High $>$ Low & Openness & Yes, $F_{(2,290)}=5.41, p^{* *}<0.01, \mathrm{C}>\mathrm{A}\left(p^{*}<0.05\right)$ \\
\hline & 2e & High $>$ Low & Accuracy & Yes, $F_{(2,290)}=4.08, p^{*}<0.05, \mathrm{C}>\mathrm{A}\left(p^{*}<0.05\right)$ \\
\hline & $2 \mathrm{f}$ & High $>$ Low & Richness & Yes, $F_{(2,290)}=3.53, p^{*}<0.05, \mathrm{C}>\mathrm{A}\left(p^{*}<0.05\right)$ \\
\hline & $2 g$ & High $>$ Low & Discussion quality & Yes, $F_{(2,290)}=3.65, p^{*}<0.05, \mathrm{C}>\mathrm{A}\left(p^{+}<0.1\right), \mathrm{C}>\mathrm{B}\left(p^{+}<0.1\right)$ \\
\hline
\end{tabular}


Table 5

Factor Mean Score Across Social Interaction Conditions for Measure of Knowledge Transfer

\begin{tabular}{|c|c|c|c|c|c|c|c|c|}
\hline Social interaction & Diversity & Assessment & Assimilation & Application & Openness & Accuracy & Richness & Quality \\
\hline \multirow{3}{*}{ Low } & Single & -0.98 & -1.60 & -1.78 & -0.80 & -0.98 & -0.72 & -0.12 \\
\hline & Multiple & -0.33 & -0.47 & 0.00 & -0.45 & -0.33 & 0.62 & 0.28 \\
\hline & Total & -0.63 & -0.99 & -0.82 & -0.61 & -0.63 & 0.00 & 0.09 \\
\hline \multirow{3}{*}{ Moderate } & Single & -2.24 & -0.50 & -1.34 & -0.55 & -1.16 & -0.29 & -0.74 \\
\hline & Multiple & 0.94 & 0.35 & -0.88 & 0.51 & 0.18 & 2.04 & 0.65 \\
\hline & Total & -0.42 & -0.01 & -1.08 & 0.06 & -0.39 & 1.04 & 0.06 \\
\hline \multirow{3}{*}{ High } & Single & 0.37 & 0.67 & -0.75 & 0.57 & 0.35 & 1.00 & 0.69 \\
\hline & Multiple & 1.06 & 1.46 & 0.19 & 1.87 & 0.85 & 2.31 & 2.06 \\
\hline & Total & 0.71 & 1.05 & -0.29 & 1.20 & 0.60 & 1.64 & 1.35 \\
\hline \multirow{3}{*}{ Total } & Single & -0.83 & -0.47 & -1.28 & -0.23 & -0.54 & 0.03 & 0.01 \\
\hline & Multiple & 0.51 & 0.39 & -0.23 & 0.57 & 0.20 & 1.60 & 0.94 \\
\hline & Total & -0.12 & -0.01 & -0.72 & 0.20 & -0.15 & 0.86 & 0.50 \\
\hline
\end{tabular}

Absorptive Capacity

Assessment. The two-way ANOVA procedure conducted on assessment scale revealed a statistically significant main effect on language diversity (H1a) $\left(F_{(1,290)}=10.58 ; p^{* *}<0.01\right)$ and social interaction (H2a) $\left(F_{(2,290)}=3.93 ; p^{*}<0.05\right)$ and a significant interaction effect between language diversity and social interaction $\left(F_{(2,290)}=3.08 ; p^{*}<0.05\right)$. Tukey's post-hoc test indicated a significant difference between a high level of social interaction (C) and a low level of social interaction (A), supporting H2a (C > A; $\left.p^{*}<0.05\right)$. When comparing with a medium level of social interaction (B), Tukey's post-hoc test did not exhibit significant differences with the high level or low level of social interaction. Under a medium level of social interaction, a great contrast at mean difference (see Figure 2) showed that communicating in multiple languages better perform assessment capacity than in a single LF.

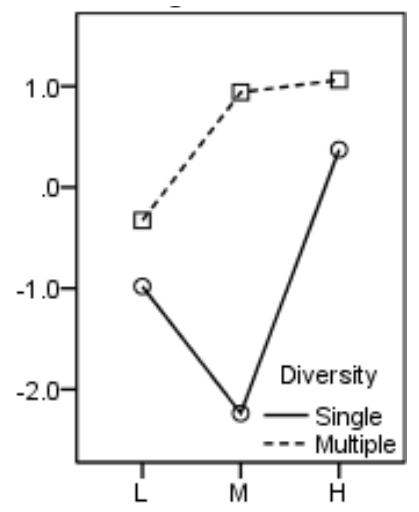

Figure 2. Assessment.

Assimilation. The two-way ANOVA procedure for assimilation scale disclosed a statistically significant main effect on language diversity (H1b) $\left(F_{(1,290)}=3.91 ; p^{*}<0.05\right)$ and social interaction $(\mathrm{H} 2 \mathrm{~b})\left(F_{(2,290)}=7.07\right.$; $\left.p^{* *}<0.01\right)$. There were no significant interaction effects between language diversity and social interaction. As predicted in $\mathrm{H} 1 \mathrm{~b}$, the results indicated that communication in multiple languages can better perform assimilation than in a single LF. A significantly result in Tukey's post-hoc test also supported H2b that teams with a high level of social interaction better performed assimilation than teams with a low level of social interaction $\left(\mathrm{C}>\mathrm{A} ; p^{*}<0.05\right)$, but no significant differences were found when compared with a medium level 
of social interaction (B). Figure 3 demonstrates the impact of language diversity and social interaction conditions on assimilation capacity.

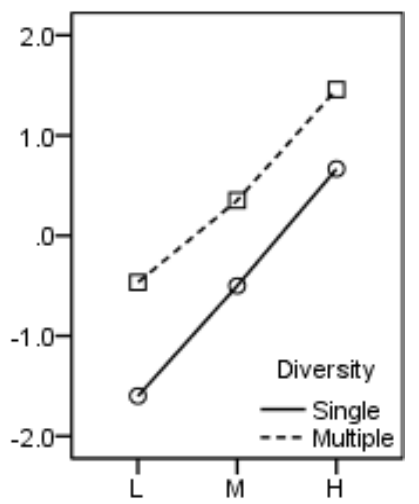

Figure 3. Assimilation.

Application. The two-way ANOVA procedure conducted for application disclosed a significant main effect for the language $\left(F_{(1,290)}=4.85 ; p^{*}<0.05\right)$, supporting H1c. Importantly, the conditions of social interaction did not affect the performance of application (H2c). The results showed no significant interaction effects between social interaction and language diversity. Figure 4 displays that teams with a medium level of social interaction and communicating in multiple languages enhanced the least capacity in application than other conditions of social interaction. Moreover, the mean difference between a single LF and multiple languages under the condition of a medium level of social interaction appeared relatively much lower than under a low level of social interaction.

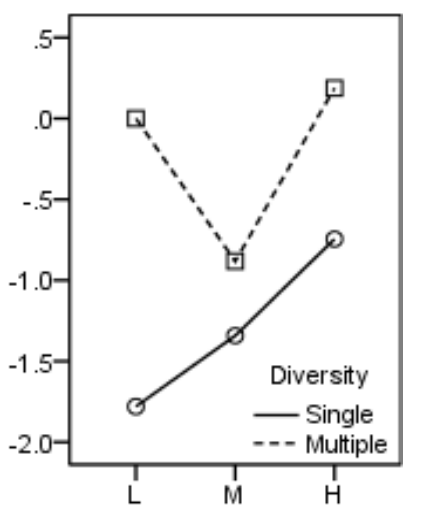

Figure 4. Application.

\section{Communication}

Openness. The two-way ANOVA procedure conducted for communication openness found a significant main effect for the conditions of social interaction $\left(F_{(2,290)}=5.41 ; p^{* *}<0.01\right)$, supporting $\mathrm{H} 2 \mathrm{~d}$, and a marginally significant difference $\left(F_{(1,290)}=3.59 ; p^{+}<0.1\right)$ on language diversity (H1d). The main effect variables were not modified by any significant interaction effect. A significantly result in Tukey's post-hoc test supported H2d that teams with a high level of social interaction performed greater communication openness than those teams with a low level of social interaction $\left(\mathrm{C}>\mathrm{A} ; p^{*}<0.05\right)$, but no significant differences were found when compared with a medium level of social interaction (B). Figure 5 demonstrates the impact of language diversity and social interaction conditions on communication openness. 


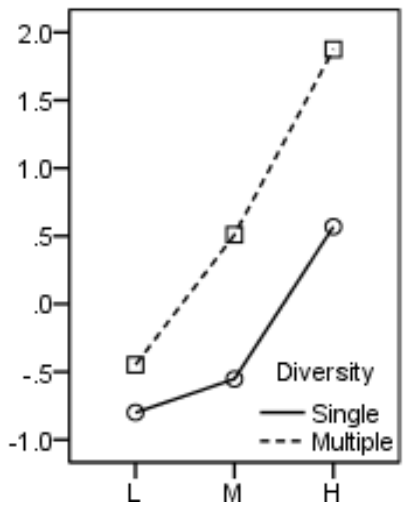

Figure 5. Opennness.

Accuracy. The two-way ANOVA procedure conducted for communication accuracy revealed significant effects of language diversity $(\mathrm{H} 1 \mathrm{e})\left(F_{(1,290)}=4.38 ; p^{*}<0.05\right)$ and conditions of social interaction (H2e) $\left(F_{(2,290)}=4.08 ; p^{*}<0.05\right)$. The interaction effects between language diversity and conditions of social interaction revealed no significant results. As predicted in H1e, the results disclosed that teams communicating in multiple languages provide greater accuracies than those teams communicating in a single LF. Tukey's post-hoc results also supported H2e, a high level of social interaction having significantly more accurate communication than a low level of social interaction ( $\left.\mathrm{C}>\mathrm{A} ; p^{*}<0.05\right)$. However, a medium level of social interaction (B) was not significantly different from either of the other levels of social interaction. Figure 6 shows that communication accuracy conducting in a single LF in a medium level of social interaction appeared the least accurate and had a highest contrast from multiple languages.

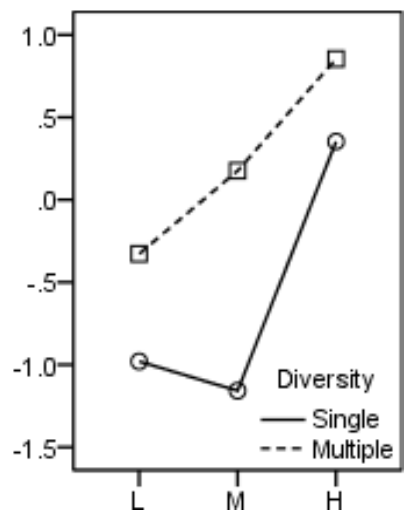

Figure 6. Accuracy.

Communication richness. The two-way ANOVA procedure conducted for communication richness disclosed significant effects for language diversity $(\mathrm{H} 1 \mathrm{f})\left(\mathrm{F}(1,290)=9.42 ; p^{*}<0.05\right)$ and conditions of social interaction $(\mathrm{H} 2 \mathrm{f})\left(\mathrm{F}(2,290)=3.53 ; p^{*}<0.05\right)$. There was not a significant interaction effect. In supporting H1f, the results indicated that the teams involving multiple languages provided richer communication than those teams communicating in a single LF. Tukey's post-hoc results also supported H2f that teams with a high level of social interaction provided richer communication at a significant level than those teams with a low level of social interaction (C > A; $p^{*}<0.05$ ). However, a medium level of social interaction (B) was not significantly different from either of the other levels of social interaction. Figure 7 demonstrates the impact of language diversity and social interaction conditions on communication richness. 


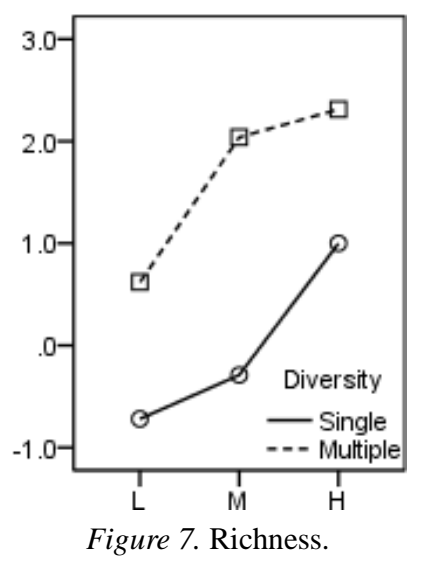

Discussion quality. The two-way ANOVA procedure conducted for discussion quality disclosed significant effects for language diversity (H1f) $\left(F_{(1,290)}=4.88 ; p^{*}<0.05\right)$ and conditions of social interaction (H2f) $\left(F_{(2,290)}=3.65 ; p^{*}<0.05\right)$. The interaction effects between language diversity and conditions of social interaction revealed no significant results. Tukey's post-hoc results indicated that a high level of social interaction had a marginally significant $\left(p^{+}<0.1\right)$ impact on discussion quality than a medium level of social interaction $(\mathrm{C}>\mathrm{B} ; p=0.075)$ and a low level of social interaction $(\mathrm{C}>\mathrm{A} ; p=0.068)$. The results of discussion quality (see Figure 8) indicated that teams communicating in a single LF performed worse than those teams communicating in multiple languages. When the communication was conducted in a single LF, the mean showed that the teams with a medium level of social interaction provided the least discussion quality. Figure 8 demonstrates the impact of language diversity and social interaction conditions on team discussion quality.

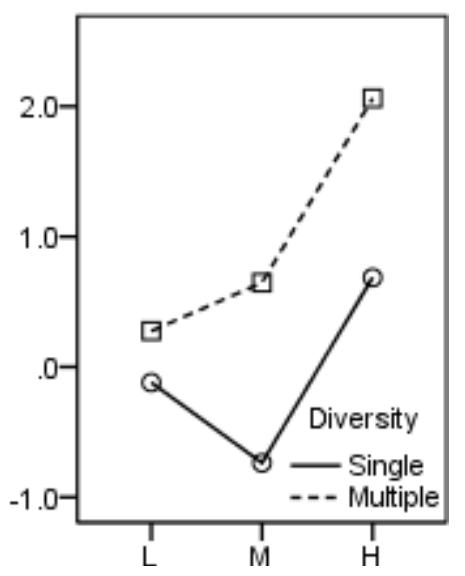

Figure 8. Discussion quality.

\section{Discussion}

This study contributes to the expanding body of research and literature on the determinants of knowledge transfer within MNCs. The main goal of this study was to address the lack of research on the impact of language diversity and social interaction on absorptive capacity and communication antecedents of the knowledge transfer process. The predictions of this study received mixed support. A key finding in the study supported the majority of the hypotheses and reinforced the view that the condition of social interaction and the language diversity through choices affect product design and service sales teams when transferring knowledge. 
Teams with a high level of social interaction communicating in multiple-choice languages can best facilitate the movement of knowledge. The results of language diversity support the concept of language impact in international management (e.g., Luo \& Shenkar, 2006; Vaara et al., 2005) that multiple languages pragmatically perform the breadth and intensity to better knowledge transfer. The complexity of physical proximity echoes early social theories (Short et al., 1976; Kock, 2004) and media theories (Carlile, 2004) that a high level of social interaction manifests synchrony and bandwidth on the physical presence to enable team actors to perceive one another (Noorderhaven \& Harzing, 2008; Watson-Manheim \& Bélanger, 2007).

With the language diversity hypotheses, the study showed significant differences for every hypothesis except communication openness between the choices of communicating in a single LF and multiple languages. Teams utilizing multiple languages can significantly have better communication in terms of accuracy, richness, and discussion quality, as well as enhance capacity in assessment, assimilation, and application than those communicating in a single LF. Involving multiple languages in a dialogue enhances a higher level of breadth and intensity (Luo \& Shenkar, 2006) to manifest the tacit dimension of social knowledge and explicit dimension of operational knowledge simultaneously. The findings may reflect that multiple languages allow team actors to enhance their capacity so as to provide contingencies to identify social, functional, or operational types of knowledge. Hence, involving multiple languages may allow team actors to identify pragmatic purposes in terms of sense making in social aspects, corporate policy in operation, or functional specialty without the need for translation, while developing ideas and integrating inter-related field knowledge. This responds to the multi-dimensional information through multiple languages that allow team actors to emphasize language characteristics by exchanging pertinent and rich information intensively, and by enhancing breadth from an individual to a group for knowledge synergy.

With the condition of social interaction hypotheses, significant differences were found for every hypothesis except for the capacity of application. A high level of social interaction can significantly provide capacity in assessment and assimilation as well as better facilitate team communication openness, accuracy, and richness. The finding may indicate that lean CMC reduces the bandwidth and synchrony to obstruct awareness of presence and affect how actors perceive and judge implicit subtleties. In contrast, teams with a high level of social interaction did not significantly perform application capacity better than those teams with other conditions of social interaction. These findings reflect that a high level of social interaction provides synchrony and bandwidth (Noorderhaven \& Harzing, 2009) for parallel and simultaneous information to enhance the capacity in assessment and assimilation, but the advantage of synchrony and bandwidth does not enhance capacity in application. This result should not be surprising because the knowledge of application in product design and sales service teams can be associated with functional specialty, i.e., computer programming language, database, and interface design, thereby, the condition of physical proximity has no direct impact on exploiting the new knowledge.

Although the level of social interaction disclosed a significant impact on discussion quality, teams with a high level of social interaction have marginally higher discussion quality than the teams with a medium level and a low level of social interaction. The finding of discussion quality manifests a salient difference of group awareness through physical presence in the F2F setting. However, the finding of discussion quality also revealed that the teams with a medium level of social interaction have no significant differences from the teams with a low level of social interaction, manifesting de-individualization in a virtual setting. This may reflect that 
the virtual setting diminishes the group awareness of relating to others, despite the social presence differences between rich CMC and lean CMC.

Teams communicating through a medium level of social interaction overall do not transfer knowledge significantly worse than those teams with a high level of social interaction. This finding may highlight the flexibility of CMC across geographical boundaries to maintain the reciprocity of retrievable information or knowledge exchanges while the social presence is compromised. While teams with a medium level of social interaction do not significantly better perform than those teams with a low level of social interaction, this finding may indicate that transmitting presentable and amendable information in lean media supplements the compromise of social presence in a virtual setting.

A joint act of multiple language choice and a high level of social interaction have a significant interaction impact on assessment capacity. When the condition of social interaction is in a virtual setting, a key contrast difference on assessment capacity shows that communicating in a single LF performs significantly worse than communicating in multiple languages. This finding may reflect that a single LF mitigates the language breadth and intensity and leads teams in a disadvantageous position. A high contrast difference of language diversity under the condition of a medium level of social interaction may suggest that communicating in multiple languages increases symbolic cues and enhance tacit dimension, i.e., social indicator, emotional cues, etc., enabling team actors in a multilingual community to associate contextual indication as a form of social cues. The situation of communication richness under the condition of a medium level of social interaction echoed that communicating in multiple languages provides richer communication than communicating in a single LF.

When teams communicate in a single LF to manage capacity in assessment, communication accuracy, and discussion quality, they can better perform under the condition of a low level of social interaction than under the condition of a medium level of social interaction. This finding may indicate that the characteristic of asynchronicity at a low level of social interaction can enhance the breadth of language and improve the process of retrieving information and gathering knowledge from different sources and activities. In general, teams with a high level of social interaction communicating in a single LF can better perform than the teams with a medium level of social interaction communicating in multiple languages. This finding may reflect the characteristics of bandwidth and synchrony provided by a high level of social interaction that may supplement the lack of language breadth and intensity when communicating in a single LF.

\section{Conclusion and Implications for Research and for Practice}

The impacts of language diversity and social interaction in MNCs provide a springboard for integrating some of the diverse research on knowledge transfer at the team level. This research shows how variations in social interaction and language diversity in foreign subsidiaries affect absorptive capacity (assessment, assimilation, and application) and communication (openness, accuracy, richness, and discussion quality). Past research has suggested the links that the role of language is a gap due to the complex in MNC structure (Luo \& Shenkar, 2006) and social interaction enhances the ability to develop social construction of knowledge (Noorderhaven \& Harzing, 2009). This study provides an empirical examination of some previous theories propositions, such as whether a multiple language design and a high level of social interaction significantly affect knowledge transfer in MNCs.

The primary contribution of this research is that this study focused on knowledge transfer within the context of MNC's operating in emerging markets in Southeast Asia and separated the role of language from 
culture as a cultural output. When the language distance of a local or national language is relatively higher than their corporate LF (West \& Graham, 2004; Dow \& Karunaratna, 2006), the use of a corporate LF to operate organizational work may be insufficient to articulate the subtleties of social indicators. Hence, the role of language complicates the process of knowledge transfer. When the knowledge of a particular language exceeds that of a single LF, this study suggests that teams utilize multiple languages to amplify language characteristics by distinguishing the differences of social subtleties and work operation. This study manifests that a single LF did not benefit knowledge transfer even though the team actors are under the impact of colonization on language. While using multiple languages can better perform knowledge transfer than using a single LF, the author proposes that team actors in a multilingual community switch between languages to improve the process of knowledge transferring.

According to an insignificant result in communication openness at language diversity, it indicates that language diversity in communication openness is contrary to the prediction. The findings may indicate that it is irrelevant between communication openness and choice of language. This finding is practically significant for any organization attempting to apply multiple languages to encourage the exchanges of interests as openness and may suggest that team actors can be just as open in a single corporate LF as in multiple languages.

The findings suggest that those product design and sales service teams in formerly colonized countries of Southeast Asia can promote constructive debate through multiple languages to strengthen the process of knowledge transfer. This research also examines the level of social interaction by the condition of social presence and manifests that those teams interacting F2F with the combination of CMC can transfer knowledge effectively, especially when combining multiple languages in a high level of social interaction reinforces the aspect of assessment capacity. However, an insignificant result of social interaction in application and a marginal impact on discussion quality presents another practical finding for organizations utilizing virtual teams, because it means that team actors can integrate knowledge as interrelated field of knowledge in a virtual setting as in a F2F setting. In particular, in a virtual setting, communicating in multiple languages reinforces the subtleties of social aspects when team actors share a common social-cultural background.

\section{Limitations and Future Study}

This study has several limitations that should be noted. First, since the type of team is product design and sales service team, the author is unaware if these results represent other team types, such as routine work teams in manufacturing production or virtual teams with geographically, organizationally, and temporally dispersed knowledge workers. The multilingual environment in this research is based in formerly colonized countries. This study is limited to make definitive conclusions if these results apply to other multilingual countries, i.e., Belgium, Switzerland, or Finland, or teams involving diverse nationalities. This study focused on a knowledge-intensive and dynamic IT industry, which limits to generalize if the findings can be applicable to other industries.

Another critical concept was how language diversity and social interaction influence knowledge transfer. The variable of language capability may affect the communication media when team actors' native languages are very different from their corporate LF. While individual capability to a common corporate LF associates the circumstance of that language choice as a foreign language, communicating through common language(s) can influence how team actors exchange pertinent information and synergize knowledge effectively. Individual 
language competence (D. E. Welch \& L. S. Welch, 2008) in communication and expertise in task operation may likely shape collective capabilities affecting knowledge transfer. Hence, language competence should separate types of capability in terms of: the capability of comprehending social-cultural subtleties while using a non-native language, the capability of processing corporate jargons for task functionality, and the capability of knowledge specialty for expertise. Several studies demonstrate that competence capability drives a great deal of intra-network communication and coordination (Cray, 1984; Nobel \& Birkinshaw, 1998; Quinn, 1996; Tsai \& Ghoshal, 1998). When examining language and social impacts in MNCs, the consideration of multi-level analysis on the input of micro-foundation of individual language competence and the output of team level of performance and macro-level of MNC performance should be further examined.

\section{References}

Ambos, T., \& Ambos, B. (2009). The impact of distance on knowledge transfer effectiveness in multinational corporations. Journal of International Management, 15(1), 1-14.

Andersen, T. J., \& Foss, N. J. (2005). Strategic opportunity and economic performance in multinational enterprises: The role and effects of information and communication technology. Journal of International Management, 11(2), 293-310.

Bandow, D. (2001). Time to create sound teamwork. Journal for Quality and Participation, 24(2), 41-47.

Barner-Rasmussen, W., \& Bjorkman, I. (2005). Surmounting interunit barriers: Factors associated with interunit communication intensity in the multinational corporation. International Studies of Management \& Organization, 35(1), 28-46.

Buckley, P. J., \& Carter, M. J. (1999). Managing cross-border complementary knowledge. International Studies of Management \& Organization, 29(1), 80-104.

Buckley, P. J., \& Carter, M. J. (2004). A formal analysis of knowledge combination in multinational enterprises. Journal of International Business Studies, 35(5), 371-384.

Burgoon, J. K., Bonito, J., Ramirez, A., Kam, K., Dunbar, N. E., \& Fischer, J. (2002). Testing the interactivity principle: Effects of mediation, propinquity, and verbal and nonverbal modalities in interpersonal interaction. Journal of Communication, 52(3), 657-677.

Cadiz, D., Griffith, T. L., \& Sawyer, J. E. (2009). Developing and validating field measurement scales for absorptive capacity and experienced community of practice. Educational and Psychological Measurement, 69(6), 1035-1058.

Carlile, P. R. (2004). A pragmatic view of knowledge and boundaries: Boundary objects in new product development. Organization Science, 13(4), 442-455.

Cohen, W. M., \& Levinthal, D. A. (1990). Absorptive capacity: A new perspective on learning and innovation. Administrative Science Quarterly, 35(1), 128-152.

Cray, D. (1984). Control and coordination in multinational corporations. Journal of International Business Studies, 15(2), 85-100.

D’Urso, S. C., \& Rains, S. A. (2008). Examining the scope of channel expansion: A test of channel expansion theory-with new and traditional communication media. Management Communication Quarterly, 21(4), 486-507.

Daft, R., Lengel, R., \& Trevino, L. (1987). Message equivocality, media selection and manager performance: Implications for information systems. Mis Quarterly, 11(3), 355-366.

Dennis, A. R., Fuller, R. M., \& Valacich, J. S. (2008). Media, tasks, and communication processes: A theory of media synchronicity. Mis Quarterly, 32(3), 575-600.

DeSanctis, G., \& Poole, M. S. (1994). Capturing the complexity in advanced technology use: Adaptive structuration theory. Organization Science, 5(2), 121-147.

Dow, D., \& Karunaratna, A. (2006). Developing a multidimensional instrument to measure psychic distance stimuli. Journal of International Business Studies, 37(5), 578-602.

Felin, T., \& Foss, N. J. (2005). Strategic organization: A field in search of micro-foundations. Strategic Organization, 3(4), 441-455.

Felin, T., Foss, N. J., Heimeriks, K. H., \& Madsen, T. L. (2012). Microfoundations of routines and capabilities: Individuals, processes, and structure. Journal of Management Studies, 49(8), 1351-1374.

Griffith, T. L., Awyer, J. E., \& Neale, M. A. (2003). Virtualness and knowledge in teams: Managing the love triangle of organizations, individuals, and information technology. Mis Quarterly, 27(2), 265-287. 
Gurtner, A., Tschan, F., Semmer, N. K., \& Nägele, C. (2007). Getting groups to develop good strategies: Effects of reflexivity interventions on team process, team performance, and shared mental models. Organizational Behavior and Human Decision Processes, 102(2), 127-142.

Kirkman, B. L., \& Mathieu, J. E. (2005). The dimensions and antecedents of team virtuality. Journal of Management, 31(5), $700-718$.

Kock, N. (2004). The psychobiological model: Towards a new theory of computer-mediated communication based on Darwinian evolution. Organization Science, 15(3), 327-348.

Lauring, J., \& Selme, J. (2012). Diversity attitudes and group knowledge processing in multicultural organizations. European Management Journal, 31(2), 124-136.

Lengel, R. H., \& Daft, R. L. (1989). The selection of communication media as an executive skill. The Academy of Management Executive, 2(3), 225-232.

Levin, D. Z., \& Cross, R. (2004). The strength of weak ties you can trust: The mediating role of trust in effective knowledge transfer. Management Science, 50(11), 1477-1490.

Lowry, P. B., Roberts, T. L., Romano, N. C., Cheney, P. D., \& Hightower, R. T. (2006). The impact of group size and social presence on small-group communication: Does computer-mediated communication make a difference? Small Group Research, 37(6), 631-661.

Luo, Y., \& Shenkar, O. (2006). The multinational corporation as a multilingual community: Language and organization in a global context. Journal of International Business Studies, 37(3), 321-339.

Mesmer-Magnus, J. R., \& DeChurch, L. A. (2009). Information sharing and team performance: A meta-analysis. Journal of Applied Psychology, 94(2), 535-546.

Minbaeva, D. B. (2007). Knowledge transfer in multinational corporations. Management International Review, 47(4), 567-593.

Mudambi, R. (2002). Knowledge management in multinational firms. Journal of International Management, 8(1), 1-9.

Nobel, R., \& Birkinshaw, J. (1998). Innovation in multinational corporations: Control and communication patterns in international R\&D operation. Strategic Management Journal, 19(5), 479-496.

Nonaka, I., \& Takeuchi, H. (1995). The knowledge-creating company-How Japanese companies create the dynamics of innovation. New York, N.Y.: Oxford University Press.

Noorderhaven, N., \& Harzing, A. W. (2009). Knowledge-sharing and social interaction within MNEs. Journal of International Business Studies, 40(5), 719-741.

O'Reilly, C. A. (1982). Variations in decision makers' use of information sources: The impact of quality and accessibility of information. Academy of Management Journal, 25(4), 756-771.

Phene, A., Madhok, A., \& Liu, K. (2005). Knowledge transfer within the multinational firm. Management International Review, 45(2), 53-74.

Quinn, J. J. (1996). The role of “good conversation” in strategic control. Journal of Management Studies, 33(3), 381-394.

Rice, R. E., \& Gattiker, U. E. (2001). New media and organizational structuring. In F. M. Jablin, \& L. L. Putnam (Eds.), The new handbook of organizational communication (pp. 544-581). Thousand Oaks, C.A.: Sage.

Roberts, T. L., Cheney, P., Sweeney, P., \& Hightower, R. (2005). The effects of information technology project complexity on group interaction. Journal of Management Information Systems, 21(3), 223-247.

Short, J., Williams, E., \& Christie, B. (1976). The social psychology of telecommunications. London, U.K.: John Wiley.

Strubler, D. C., \& York, K. M. (2007). An exploratory study of the team characteristics: Model using organizational teams. Small Group Research, 38(6), 670-695.

Szulanski, G. (1996). Exploring internal stickiness: Impediments to the transfer of best practice within the firm. Strategic Management Journal, 17(Winter Special Issue), 27-43.

Thompson, L. L. (2011). Sharpening the team mind: Communication and collective intelligence. In Making the team: A guide for managers (4th ed.). Upper Saddle River, N.J.: Prentice Hall.

Tidwell, L. C., \& Walther, J. B. (2002). Computer-mediated communication effects on disclosure, impressions, and interpersonal evaluations: Getting to know one another a bit at a time. Human Communication Research, 28(3), 317-348.

Tiwana, A., \& McLean, E. R. (2005). Expertise integration and creativity in information systems development. Journal of Management Information Systems, 22(1), 13-43.

Todorova, G., \& Durisin, B. (2007). Absorptive capacity: Valuing a reconceptualization. Academy of Management Review, 32(3), 774-786. 
Trevino, L. K., Webster, J., \& Stein, E. W. (2000). Making connections: Complementary influences on communication media choices, attitudes, and use. Organization Science, 11(3), 163-182.

Tsai, W., \& Ghoshal, S. (1998). Social capital and value creation: The role of intrafirm networks. Academy of Management Journal, 41(4), 464-476.

Vaara, E., Tienari, J., \& Piekkari, R. (2005). Language and the circuits of power in a merging multinational corporation. Journal of Management Studies, 42(3), 595-623.

Watson-Manheim, M. B., \& Bélanger, F. (2007). Communication media repertoires: Dealing with the multiplicity of media choices. Mis Quarterly, 31(2), 267-293.

Welch, D. E., \& Welch, L. S. (2008). The importance of language in international knowledge transfer. Management International Review, 48(3), 339-360.

West, J., \& Graham, J. L. (2004). A linguistic-based measure of cultural distance and its relationship to managerial values. Management International Review, 44(3), 239-260.

Zahra, S. A., \& George, G. (2002). Absorptive capacity: A review, reconceptualization, and extension. Academy of Management Review, 27(2), 185-203. 


\section{Appendix: Knowledge Transfer Survey Scales}

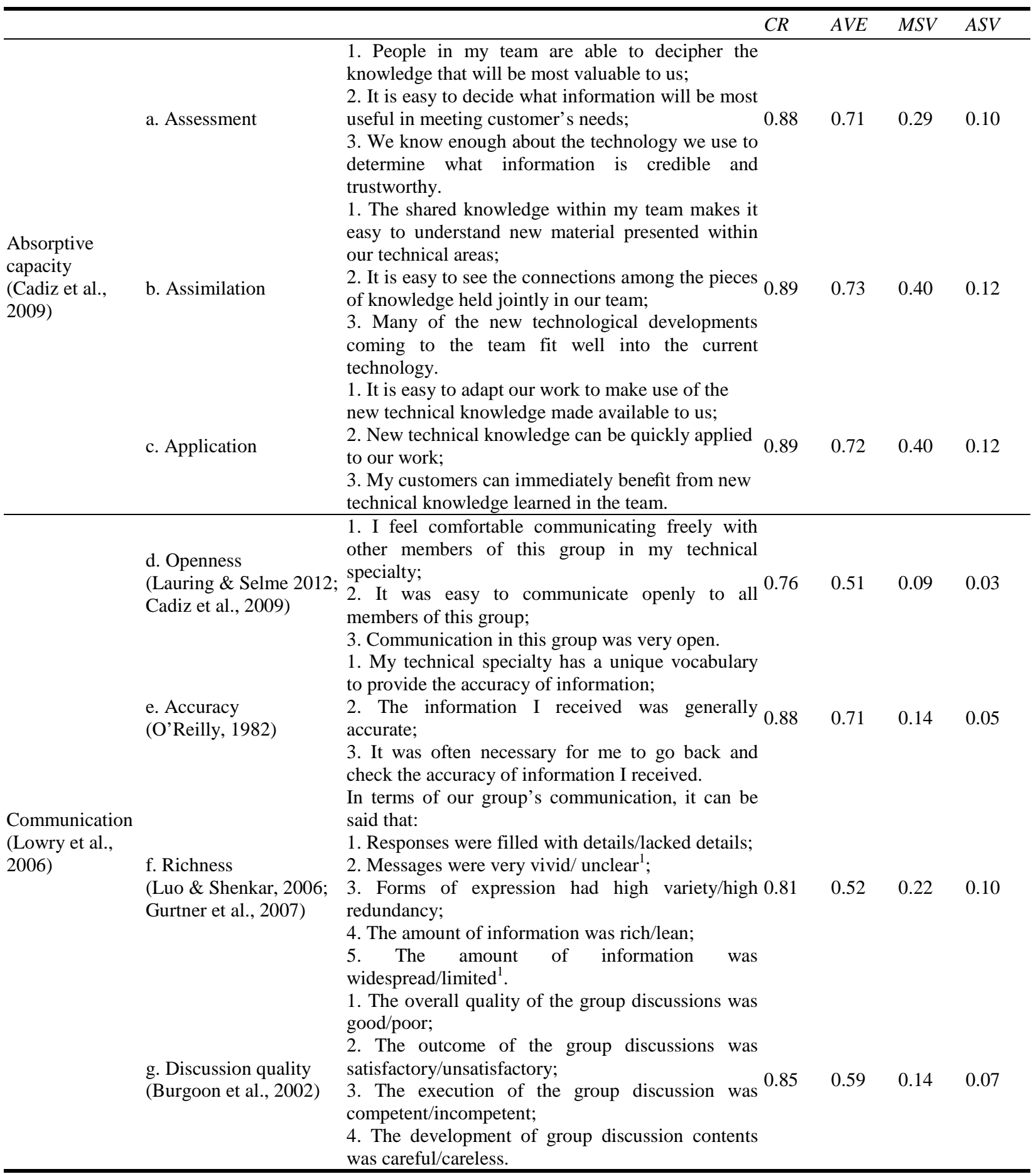

Notes. $C R=$ Composite reliabilities, $A V E=$ Average variance extracted, $M S V=$ Maximum shared variance, and ASV $=$ Average shared variance; Items from "a” to "e" were on a scale of 1 (Negative) to 7 (Positive); ${ }^{1}$ Deleted item. 